\title{
Study on Vibration and Noise Characteristics of Pure Electric Bus
}

\author{
Hongfei Yang ${ }^{1}$, Congze $\mathrm{Lin}^{2}$, Wei $\mathrm{Li}^{3}$ \\ ${ }^{1}$ School of Power and Mechanical Engineering, Wuhan University, Wuhan, Hubei, China \\ ${ }^{2}$ Xiamen University Malaysia, Selangor, Malaysia \\ ${ }^{3}$ North China Electric Power University (Baoding), Baoding, Hebei, China
}

Keywords: vehicle engineering; pure electric bus; vibration and noise; order tracking; motor excitation.

\begin{abstract}
In this paper, an experimental study is carried out on the vibration and noise of a pure electric bus under acceleration and uniform speed conditions. The vibration and noise data in the vehicle are collected by LMS sound and vibration signal acquisition system, and the vibration and noise excitation sources are analyzed by means of frequency spectrum analysis and order tracking, and the vibration and noise characteristics of electric vehicles are summarized. The experimental results show that the vibration in the vehicle at constant speed is mainly the road excitation from the suspension, and the excitation of the motor is more obvious when driving at an accelerated speed, especially the high frequency excitation near the switching frequency of the motor. The study in this paper can provide a basis for the improvement of vibration and noise of electric vehicles in the next step.
\end{abstract}

\section{Introduction}

Compared with traditional cars with internal combustion engines, electric vehicles are only powered by motors. People often think that the vibration and noise of electric vehicles will be very small, but due to the disappearance of the "masking effect" of the engine, many vibration and noise problems that are not easy to find in traditional vehicles are highlighted, such as fan noise, gear meshing vibration noise, motor electromagnetic vibration noise and so on[1].

In recent years, many scholars at home and abroad have studied the vibration and noise of hybrid electric vehicles and pure electric vehicles. Pellerey and others carry on the magnetic-solid coupling simulation analysis of the power transmission system of the pure electric vehicle, which proves that the current harmonic of the vehicle permanent magnet synchronous motor has a strong effect on the vibration and noise. Shin et al tested and improved the noise of a new type of electric vehicle, and found that the noise of electric vehicle is lower than that of the same type of internal combustion engine vehicle $(10 \mathrm{~dB}(\mathrm{~A})$,), but the high frequency noise of electric vehicle is obvious. Wang and others believe that the torque fluctuation of the electric vehicle motor will cause the low speed jitter of the whole vehicle, especially when the frequency of the fluctuation is close to the resonant frequency of the transmission system. Yan Gang and others carried out experimental research on the interior noise of a pure electric vehicle and analyzed the contribution of driving motor, reducer and battery cooling fan to the interior noise. Huang Xiaoci and others analyzed the noise sources of hybrid electric vehicles under pure electric and hybrid conditions, and concluded that compound planetary gear meshing is the main noise source in pure electric mode. Fangyuan and others carried out the bench test on the centralized drive electric vehicle, analyzed the vibration and noise characteristics of the powertrain under the positive drive condition and the reverse drag condition, and concluded that the electromagnetic noise and gear howling are the main noise sources of the electric vehicle; at low speed, the noise generated by the powertrain and auxiliary system is the main source of noise outside the vehicle. Zhu Yu established the prediction model of the sound quality of the pure electric vehicle by using the neural network method, and analyzed the influence of the driving motor on the sound 
quality in the vehicle. Guo Rong and others identify the main noise source of the fuel cell car and determine that the noise source is the fuel cell auxiliary system[2].

In this paper, taking a pure electric bus as the research object, the road condition test of the vibration and noise of the whole vehicle is carried out, and the vibration and noise characteristics of the driver and the rear seat are analyzed. the main excitation sources of vibration and noise in the vehicle under uniform speed and accelerated driving conditions are obtained. The research in this paper can provide a basis for the next step to improve the NVH performance of the whole vehicle.

\section{Test vehicle}

The test car is a medium passenger car with a length of $6 \mathrm{~m}$ and a wheelbase of $3.3 \mathrm{~m}$. The no-load mass is $3650 \mathrm{~kg}$, the full load is $5200 \mathrm{~kg}$, and the number of seats is 17 (including drivers). The driving motor is permanent magnet synchronous motor and the peak power is $100 \mathrm{~kW}$. The battery is lithium iron phosphate battery, 336V and 200A.h.

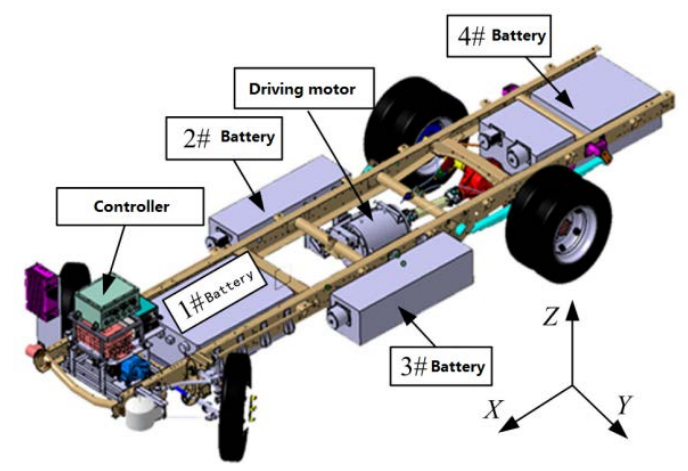

Fig 1 Vehicle structure drawing

Figure 1 is the structure diagram of the electric vehicle, which is equipped with four power batteries, which are evenly arranged on the frame. The motor is arranged in the middle of the chassis, and the motor output shaft transmits power to the rear axle through the transmission shaft. Fig. 1 the structure diagram of the whole vehicle Fig.1 Structureofelectricvehicle omits the transmission and clutch of the traditional car and adjusts speed directly through the motor[3]

\section{Test scheme}

\subsection{Test purpose}

The vibration and noise tests of the sample vehicle are carried out under the condition of acceleration and uniform speed, and the vibration and noise characteristics of the electric vehicle are analyzed, which provides a basis for the improvement of the vibration and noise of the electric vehicle in the next step.

\subsection{The location of the sensor}

Binary images can remove table lines and small noises through graphic morphological operations, and form connected domains of similar characters. Markers can surround the largest rectangle of connected domains, which can achieve the positioning of text. Fig. 8 and Fig. 9 are graphs of connected domains of text obtained after a series of morphological processing and text areas located by filtering. 


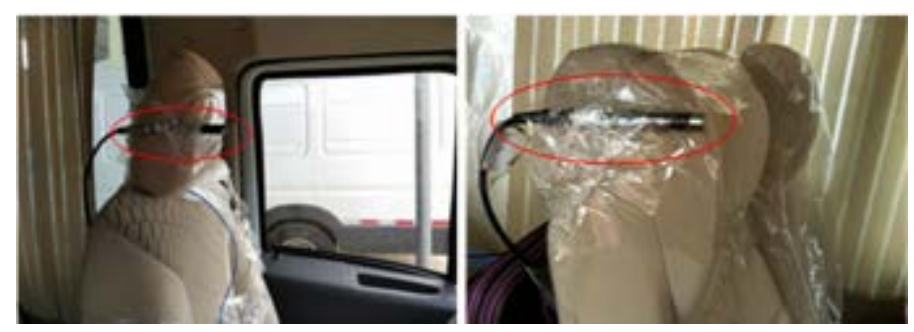

Fig 2 Acoustics the location of the Mike.
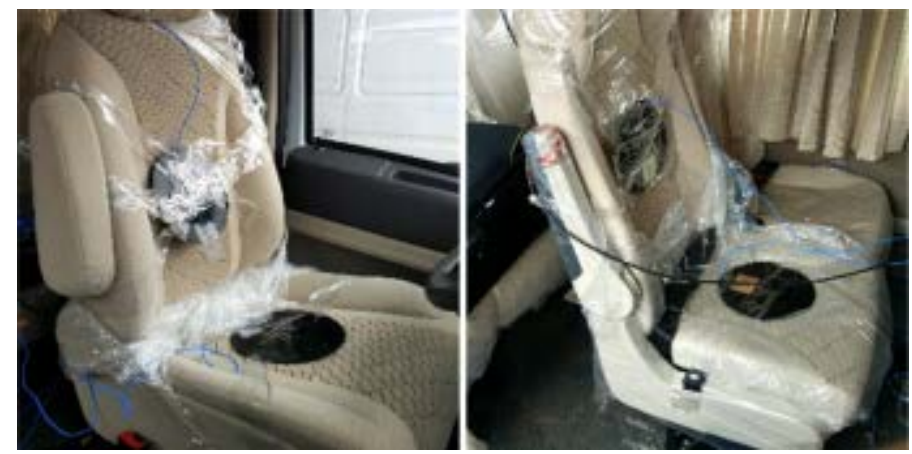

Fig 3Cushion sensor

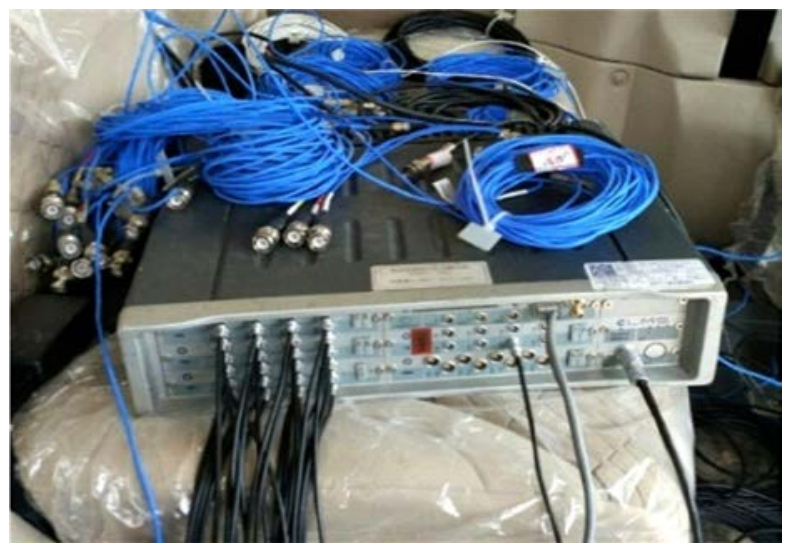

Fig 4 LMS data acquisition front end

\subsection{Test condition}

The test site is Dingyuan Automobile Test site in Anhui Province, and the road surface is class B road surface in accordance with GB7031 regulations. The contents of the experiment are as follows:

(1) bias frequency test.

(2) uniform working condition: the vehicle runs at a uniform speed of 40, 50, 60, 70, 80, 90 and $100 \mathrm{~km} / \mathrm{h}$, and the vibration and noise signal of the whole vehicle is collected, and the signal acquisition time is 20 s.

(3) acceleration condition: the vehicle accelerates rapidly from rest (throttle to the end) to the maximum speed $(100 \mathrm{~km} / \mathrm{h})$, and collects the vibration and noise signals of the whole vehicle after acceleration.

\section{Test results}

\subsection{Uniform driving condition}

\subsubsection{Driver seat vibration}

Figure 5 shows the frequency spectrum of the guide rail vibration of the driver seat, in which the seat vibration is mainly the low frequency vibration from the suspension. At all speeds, there are two maximum peaks of $1.7 \mathrm{~Hz}$ and $17 \mathrm{~Hz}$, corresponding to the spring-loaded and non-spring-loaded mass 
bias of the front suspension, respectively, and the vibration amplitudes of more than $500 \mathrm{~Hz}$ are very small and can be ignored. Therefore, when the vehicle is running at a constant speed, the vibration of the seat in the vehicle is mainly caused by the road excitation from the suspension.
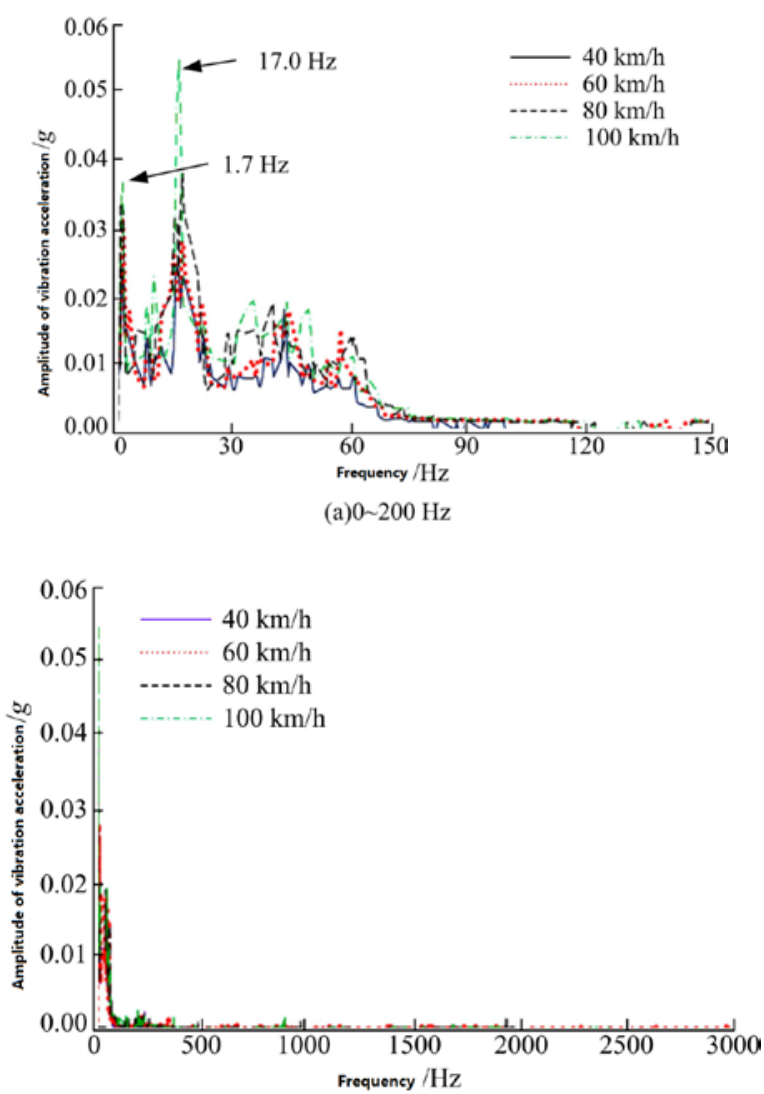

(b) $0 \sim 3000 \mathrm{~Hz}$

Fig 5 Z-direction vibration spectrum of driver's seat

\subsubsection{Driver position noise}

As can be seen from figure 6, with the increase of vehicle speed, the interior noise increases gradually, but the noise is mainly concentrated in the middle and low frequency within the $300 \mathrm{~Hz}$, and the order noise of the motor is not obvious.

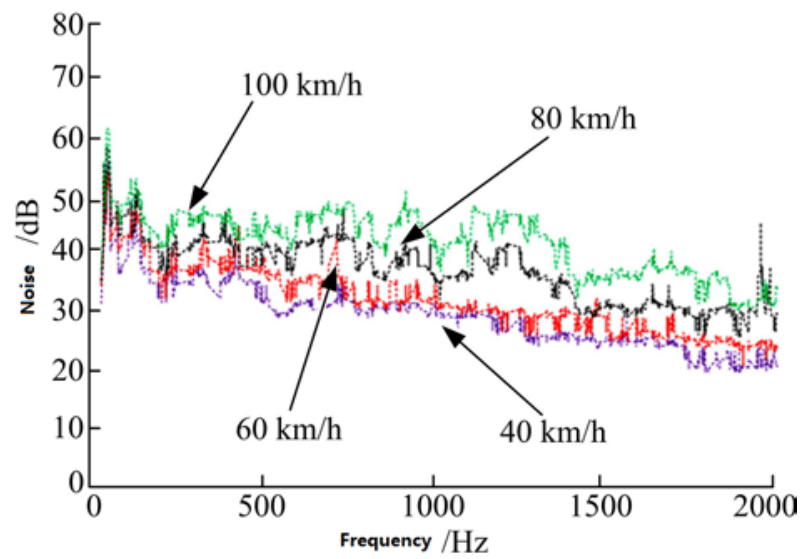

Fig 6 Noise spectrum of driver seat position 


\subsection{Frequency shift test}

Frequency bias is an important parameter of vehicle performance, which plays an important role in analyzing the road excitation from suspension. In order to accurately measure the bias frequency of the automobile front (rear) suspension, it is necessary to lock the elastic element and non-spring load mass of the rear (front) suspension to avoid the coupling of the front suspension and the rear suspension. However, compared with the spring-loaded mass, the non-spring-loaded mass is smaller, and the suspension mass distribution coefficient of most vehicles is $0.8-1.2$, so it can be considered that the vertical motion of the spring mass of the front and rear suspension is independent of each other. Combined with the conditions of the test site, the frequency bias test of the whole vehicle is carried out by using the following test method: as shown in figure 7 , during the test, the front wheel (rear wheel) of the vehicle is driven to the trapezoidal hardwood bump and stopped, and the front wheel is pushed down from the bump by manpower. the vibration curves of spring and off-spring mass are collected.

The frequency offset results of the front and rear suspension are shown in Table 2. For the spring mass bias, the front suspension is a double cross-arm independent suspension with smaller frequency offset, while the rear suspension is an integral axle with a single-stage stiffness and a multi-leaf spring structure with larger frequency offset.

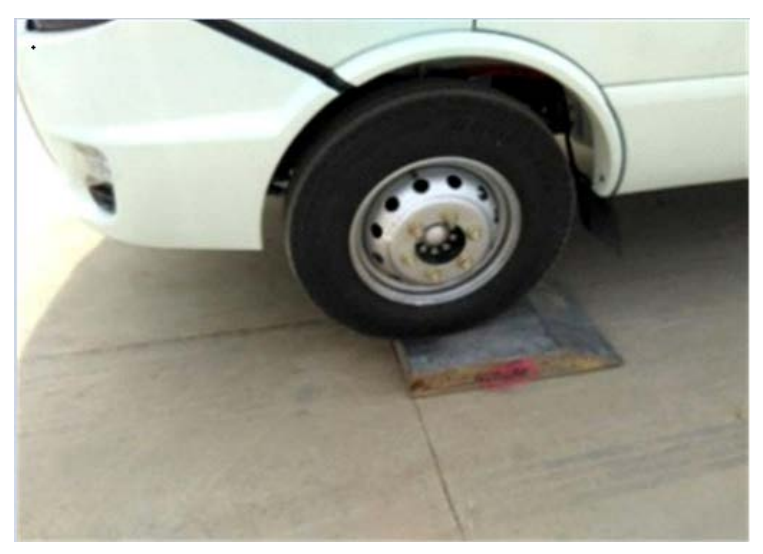

(a)front suspension

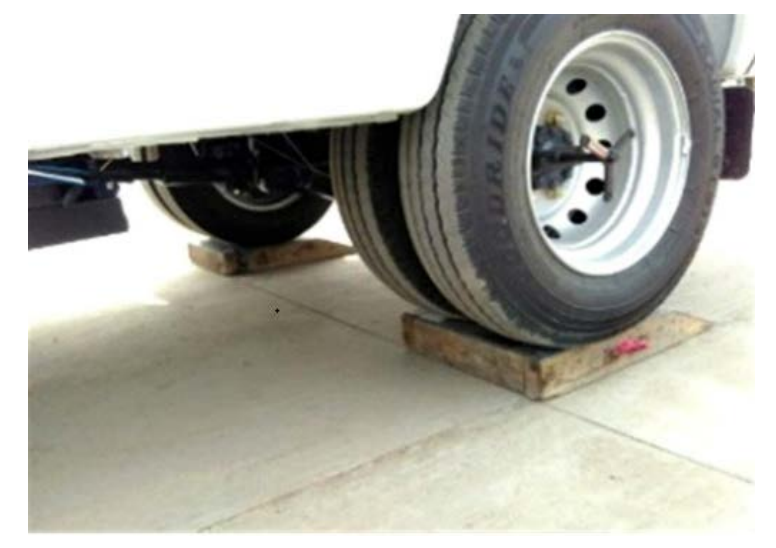

(b)rear suspension

Fig 7 Frequency shift test

The frequency offset results of the front and rear suspension are shown in Table 2. For the spring mass bias, the front suspension is a double cross-arm independent suspension with smaller frequency offset, while the rear suspension is an integral axle with a single-stage stiffness and a multi-leaf spring structure with larger frequency offset.

\subsection{Uniform acceleration driving condition}


Permanent magnet synchronous motor (PMSM) is widely used in electric vehicles because of its advantages such as large torque and small size. The parameters of the electric vehicle permanent magnet synchronous motor studied in this paper are as follows: the peak power is $100 \mathrm{~kW}$, the maximum speed is $4000 \mathrm{rpmin}$, the peak torque is $1000 \mathrm{~N} \mathrm{~nm}$, the pole number of the rotor is 4 , the number of stator slots is 48 , and the switching frequency of the inverter is $4000 \mathrm{~Hz}$.

Under the condition of power supply by frequency converter, the input voltage or current of vehicle permanent magnet synchronous motor is not an ideal sine wave, but a series of voltage or current pulses of equal amplitude and unequal width, which are decomposed by Fourier series. a series of time harmonics can be obtained, which can produce large electromagnetic force waves in the motor, and then cause the increase of motor vibration and noise.

When the vehicle is accelerating, the vibration and noise of the motor is particularly obvious.

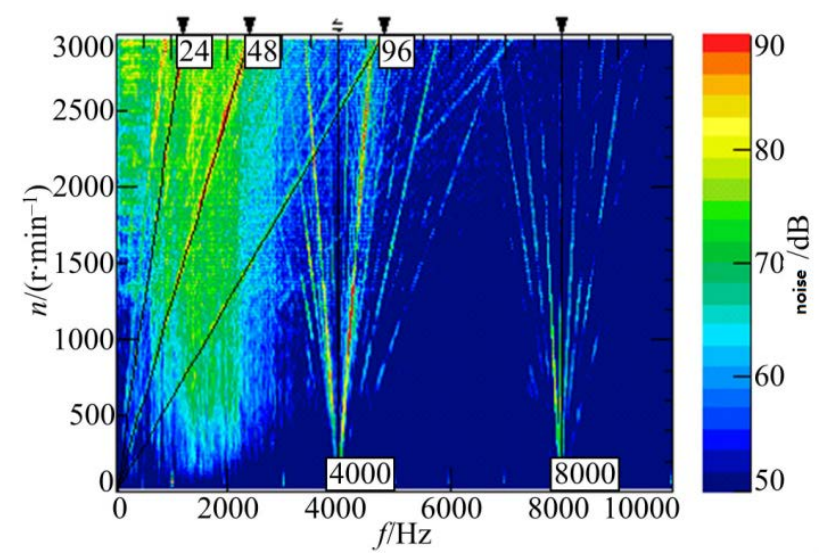

Fig 8 Order tracking diagram of motor near-field noise

In order to analyze the contribution of each order noise to the total noise, as shown in figure 8 , the order slice analysis of the noise in figure 7 is carried out. It can be seen that when the rotating speed of the motor is within $1000 \mathrm{r} / \mathrm{min}$, the noise difference of each order is small; when the speed exceeds $1000 \mathrm{r} / \mathrm{min}$, the 48th-order noise of the motor is the largest, and with the increase of speed, the difference between the 48th-order noise and the total noise gradually decreases, indicating that the contribution of each order noise to the total noise is increasing.

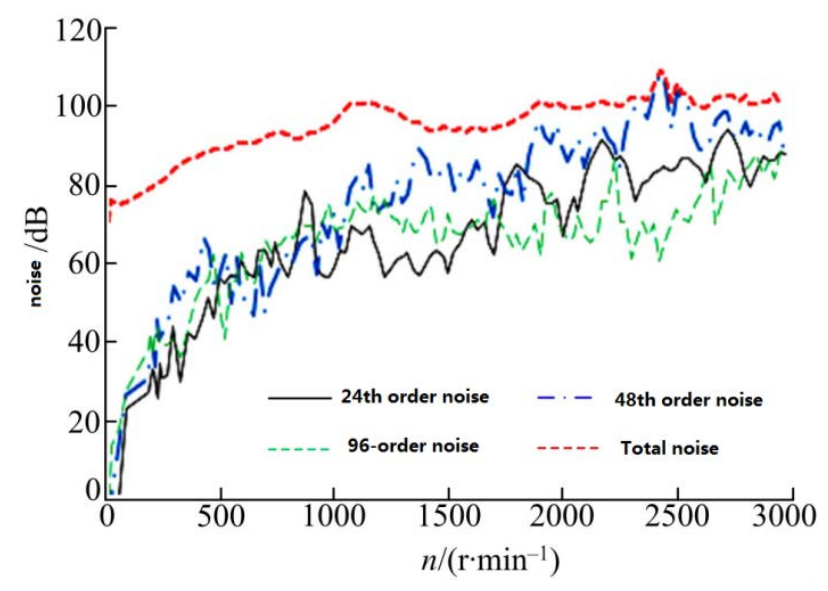

Fig 9 Order slice diagram of motor noise

In figure 8, there are many "fireworks" harmonic excitations near the inverter switching frequencies $4000 \mathrm{~Hz}$ and $8000 \mathrm{~Hz}$, which is the biggest difference between electric vehicles and internal combustion engine vehicles. This is because the permanent magnet synchronous motor 
(PMSM) provides three-way alternating current by the inverter. due to the current modulation, there will be a series of high-order harmonic components with close carrier frequency in the stator current.

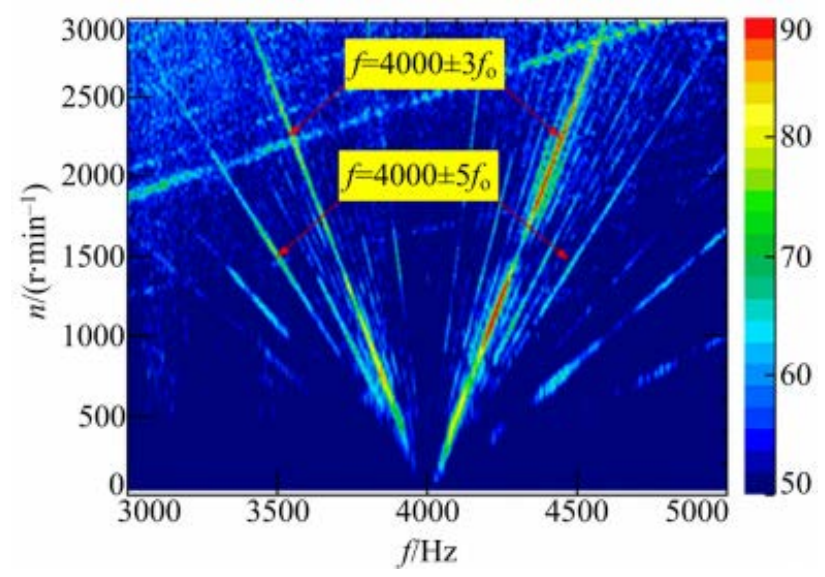

(a)Center frequency $4000 \mathrm{HZ}$

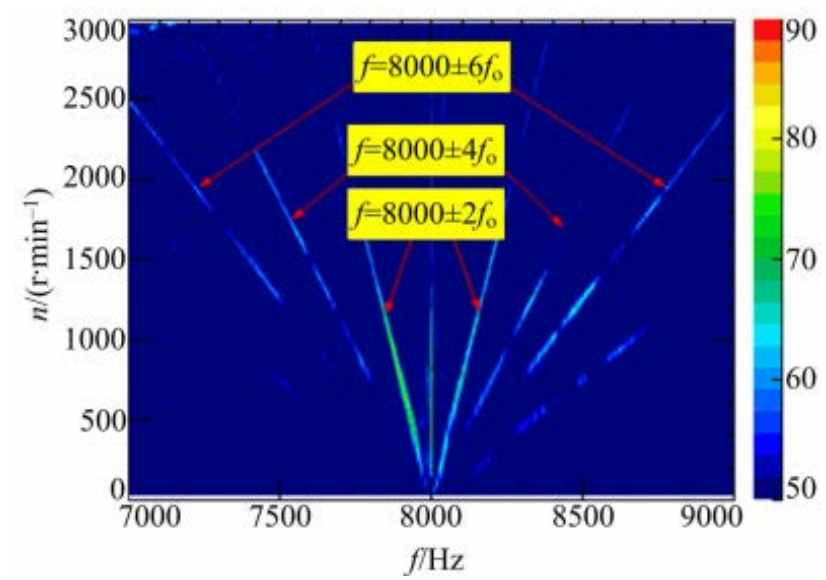

(a)Center frequency $8000 \mathrm{HZ}$

Fig 10Motor high frequency noise

Figure 11 shows the order tracking diagram of the vibration of the driver's seat, which shows that the 8th, 12th and 24th order excitation of the motor is more obvious.

In order to compare the influence of motor excitation on driver's seat vibration during constant speed and acceleration, the order tracking chart is sliced according to speed. Due to the limitation of space, only the difference between the two is analyzed when the speed is $80 \mathrm{~km} / \mathrm{h}$.

It can be seen from figure 12 that there is little difference in the frequency spectrum of driver's seat vibration between acceleration and uniform speed. The driver's seat vibration mainly comes from low-frequency road excitation, which is mainly concentrated within the $60 \mathrm{~Hz}$. 


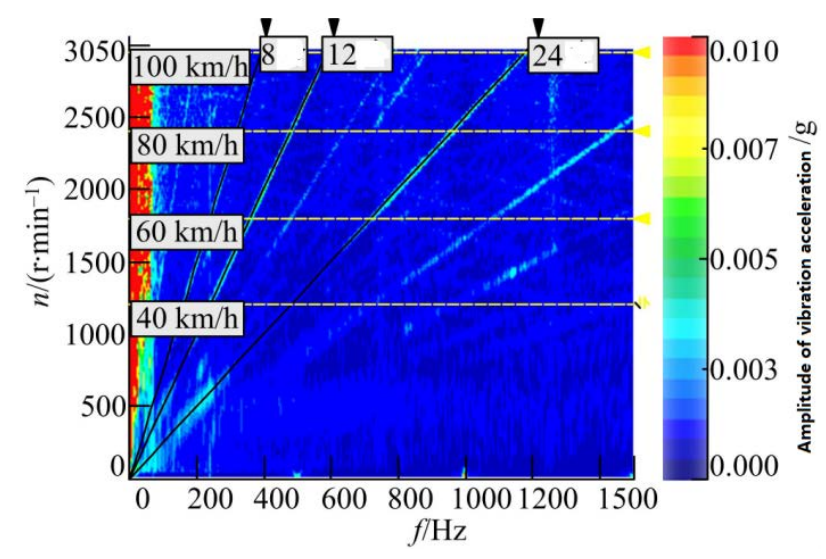

Fig 11 Order tracking diagram of driver seat vibration (z direction)

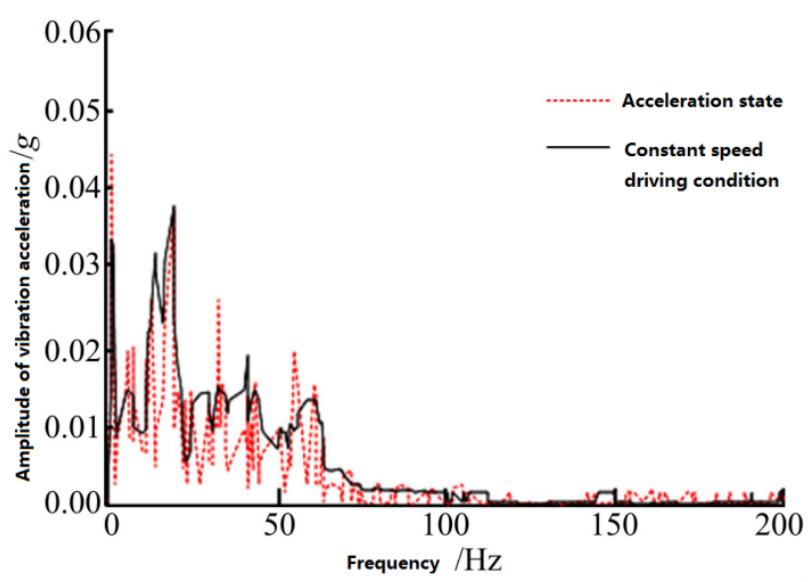

Fig 12 Driver seat Vibration (80km/h) at acceleration and uniform speed

\section{Conclusion}

When the electric bus is running at a constant speed, the vibration of the seat in the car is mainly excited by the road surface from the suspension, the frequency is low, and the frequency at the peak of the vibration basically coincides with the bias frequency of the suspension. When driving at accelerated speed, due to the shielding and sealing effect of the car body, the high frequency noise of the motor has little influence on the interior of the car, and the harmonic excitation frequency near the $4000 \mathrm{~Hz}$ in the car is $\mathrm{f}=4000 \pm 3 \mathrm{f} 0$. The motor has a great influence on the acceleration noise outside the vehicle, with high frequency and poor subjective feeling, which can increase the switching frequency of the motor and reduce the harmonic noise of the switching frequency.

\section{References}

[1] Yu Peng, he Lizhao, Zhang Tong, et al. Research status and prospect of NVH performance of vehicle powertrain driven by centralized motor [J]. Mechanical equipment Design, 2014, 31 (3): 16

[2] Qin Qin, Xiao Weimin, Jiang Congshuang, et al. Comparison of noise characteristics between electric vehicles and fuel vehicles [J]. Noise and Vibration Control, 2014

[3] Yan Gang, Xia Shunli, Zhang Huanhuan, et al. Test analysis and identification of interior noise of a pure electric car [J]. Journal of Hefei University of Technology: Zireng Science Edition, 2011

[4] Fang Yuan, Zhang Tong, Yu Peng, et al. Analysis and test of noise characteristics of centrally driven electric vehicles [J]. Vibration and impact, 2015, 34 (13): 8994 